\title{
In vitro co-culture of Solanum tuberosum hairy roots with Meloidogyne chitwoodi: structure, growth and production of volatiles
}

\author{
Jorge M. S. Faria • Inês Sena • Carla M. Maleita • \\ Inês Vieira da Silva · Lia Ascensão - Isabel Abrantes • \\ Richard N. Bennett · Manuel Mota $\cdot$ A. Cristina Figueiredo
}

Received: 2 December 2013/ Accepted: 2 May 2014

(C) Springer Science+Business Media Dordrecht 2014

\begin{abstract}
Meloidogyne spp., commonly known as rootknot nematodes (RKNs), are economically important plant sedentary endoparasites that cause galls on susceptible hosts. The Columbia root-knot nematode (CRKN), $M$. chitwoodi, is a quarantine A2 type pest by the European and Mediterranean Plant Protection Organization since 1998. This nematode has been found associated with economically important crops such as potato and tomato, causing severe damage and making the agricultural products unacceptable for the fresh market and food processing. In vitro co-culture of host and parasite offers an advantageous experimental system for studying plant-RKN interactions. The structure, growth and production of volatiles of Solanum tuberosum hairy roots (HR) and of $S$. tuberosum HR/ CRKN co-cultures were compared. HR were induced by inoculation of aseptic potato tuber segments with Rhizobium rhizogenes. Co-cultures were initiated by inoculating $\mathrm{HR}$ with sterilized CRKN eggs. Infection with CRKN induced the RKN symptomatology in the HR and several nematode life stages were observed by light and scanning electron microscopy. Potato HR and HR/CRKN co-cultures
\end{abstract}

J. M. S. Faria · I. Sena · I. Vieira da Silva · L. Ascensão

A. C. Figueiredo $(\bowtie)$

Universidade de Lisboa, Faculdade de Ciências de Lisboa, DBV, IBB, Centro de Biotecnologia Vegetal, C2, Campo Grande, 1749-016 Lisboa, Portugal, Lisbon, Portugal

e-mail: acsf@fc.ul.pt

C. M. Maleita

CIEPQPF, Departamento de Engenharia Química, Universidade de Coimbra, 3030-790 Coimbra, Portugal, 3030-790 Coimbra, Portugal

I. Abrantes

IMAR-CMA, Departamento de Ciências da Vida, Universidade de Coimbra, 3004-517 Coimbra, Portugal, Coimbra, Portugal exhibited similar growth patterns, evaluated by measuring fresh and dry weight and by the dissimilation method. Volatiles, isolated by distillation-extraction and analyzed by gas chromatography (GC) and gas chromatography coupled to mass spectrometry, revealed that palmitic acid (37-52\%), n-pentadecanal (10-16\%) and linoleic acid (2-16\%) were the main constitutive components of $S$. tuberosum HR, and of the HR/CRKN co-cultures (24-44, 8-22 and 4-18\%, respectively). S. tuberosum HR/CRKN co-cultures can be considered a suitable biotechnological tool to study RKN infection mechanism by mimicking what occurs under field conditions.

Keywords Plant biotechnology - Columbia root-knot nematode $\cdot$ Potato $\cdot$ Hairy root structure $\cdot$ Root gall structure $\cdot$ Volatiles

\section{Abbreviations \\ $\mu \quad$ Specific growth rate \\ CRKN Columbia root-knot nematode \\ (Meloidogyne chitwoodi) \\ DAI Days after inoculation}

R. N. Bennett

Universidade de Trás-os-Montes e Alto Douro, Quinta dos Prados-Apartado 1013, 5000-911 Vila Real, Portugal

M. Mota

NemaLab, ICAAM-Instituto de Ciências Agrárias e Ambientais Mediterrânicas, Universidade de Évora, Núcleo da Mitra, Ap. 94, 7002-554 Évora, Portugal

M. Mota

INIAV/Unidade Estratégica de Investigação e Serviços de Sistemas Agrários e Florestais e Sanidade Vegetal, Av. da República, Quinta do Marquês, 2784-159 Oeiras, Portugal 


$\begin{array}{ll}\text { dt } & \text { Doubling time } \\ \text { DW } & \text { Dry weight } \\ \text { EPPO } & \begin{array}{l}\text { European and Mediterranean Plant } \\ \text { Protection Organization }\end{array} \\ \text { FW } & \text { Fresh weight } \\ \text { GC } & \text { Gas chromatography } \\ \text { GC-MS } & \text { Gas chromatography coupled to } \\ & \text { Mass Spectrometry } \\ \mathrm{HgCl}_{2} & \text { Mercuric chloride } \\ \mathrm{HR} & \text { Hairy roots } \\ \mathrm{J} 2 & \text { Nematode second-stage juveniles } \\ \mathrm{LM} & \text { Light microscopy } \\ \mathrm{NaOCl} & \text { Sodium hypochlorite } \\ \mathrm{OsO} & \\ \mathrm{PAS} & \text { Osmium tetroxide } \\ \mathrm{RI} & \text { Periodic acid-Schiff's reagent } \\ \mathrm{RKN} & \text { Retention index } \\ \mathrm{SEM} & \text { Root-knot nematode } \\ \mathrm{SH} & \text { Scanning electron microscopy } \\ \mathrm{t} & \text { Schenk and Hildebrandt medium } \\ \end{array}$

\section{Introduction}

The Columbia root-knot nematode (CRKN) Meloidogyne chitwoodi Golden, O'Bannon, Santo and Finley 1980, is a sedentary and obligate plant endoparasite, which was described for the first time in the Pacific Northwest of the USA and has been reported in Africa (South Africa), Europe (Belgium, Germany, the Netherlands and Portugal) and North and South America (Mexico and Argentina, respectively) (Golden et al. 1980; da Conceição et al. 2009; OEPP/EPPO 2009). This species is a quarantine A2 type pest according to the European and Mediterranean Plant Protection Organization since 1998 (EPPO 2012) and has been found associated with economically important crops such as potato (Solanum tuberosum L.) and tomato (S. lycopersicum L.), causing severe damage responsible for heavy losses and/or unacceptable agricultural products for the fresh market and food processing (Santo et al. 1980; O’Bannon et al. 1982; Ferris et al. 1993; Perry et al. 2009).

These endoparasites mobilize the plant's photosynthates from shoots to roots and affect water and nutrient absorption and translocation by the roots to support their development and reproduction. Root-knot nematode (RKN) second-stage juveniles (J2), the infective stage, are attracted to a host plant, invade the root and move through the apoplast to the zone of differentiation. There, they induce the formation of giant cells (feeding site) and typical root galls (Perry et al. 2009). During this process, most of the J2 become sedentary and enlarge, assuming a pear shape and moult three times reaching the female adult stage and lay eggs into a gelatinous matrix produced by rectal glands. Males are vermiform and motile, leaving the roots, and are less frequent. However, the proportion increases under environmental stress conditions (Perry et al. 2009).

RKN pest management has been extensively documented (e.g., Griffin 1985; Chitwood 2002, 2003; Li et al. 2011; Andrés et al. 2012). Nevertheless, research on the effect of nematicidal compounds is commonly performed on the nematode species alone and rarely on the host-parasite system, not taking into account the cytotoxicity for the plant host or the plant's capability to metabolize or biotransform the nematicidal active substances. When studying complex in vivo organism-organism interactions, analyzing just one of the partners gives only a partial view of the existing relations. For this reason, the growth of more than one organism or cell type in a combined culture (in vitro co-cultures) has the advantage of simulating the host-pathogen conditions and eliminating variables due to the environmental in vivo conditions.

In vitro axenic culture of hairy roots (HR), or transgenic roots, offers a suitable biotechnological model host system for analysis of RKN infection, due to their genetic and metabolomic stability as well as a rapid growth rate, compared to conventional root cultures, and to being free of plant growth regulators (Giri and Narasu 2000; Figueiredo et al. 2006). In vitro plant/nematode co-cultures have been commonly used since mid-1900's to increase and maintain nematodes and study plant/nematode interactions (Bonga and Durzan 1982; Maheshwari 1991). In monoxenic cocultures of Meloidogyne spp., the host-pathogen system is free from contaminants such as soil flora and fauna which characterize the natural conditions (Bonga and Durzan 1982; Mitkowski and Abawi 2002). Moreover, in a controlled environment of an in vitro culture, single variables can be manipulated and plant/nematode responses can be observed directly, which is difficult under greenhouse or field conditions. Furthermore, in vitro cultures have the advantage of providing more biomass using fewer resources.

Although potato HR have been previously established and used for metabolomic (Valancin et al. 2013) and genetic transformation evaluation (Nagy et al. 2005), and studies have addressed nematode effect on potato hairy root cultures (Hansen et al. 1996; Wiśniewska et al. 2013), no previous study established a $S$. tuberosum $\mathrm{HR} / M$. chitwoodi co-culture system, which requires regular subculturing of the host/pathogen system.

Given that RKN quarantine species is a serious potato pest (Mojtahedi et al. 1988; Perry et al. 2009) and there is the need for a reliable host-pathogen system for phytopathological research, e.g. phytonematicidal research, the goals of this study were to: (a) establish $S$. tuberosum HR, and $S$. tuberosum HR/M. chitwoodi co-cultures ( $S$. tuberosum HR/CRKN co-cultures), (b) evaluate HR and the 
co-cultures structure and growth, (c) quantify the nematodes in in vitro co-cultures medium and (d) characterize the constitutive and induced production of volatiles in the two independent in vitro culture systems. The present study proposes that the nematode life cycle in co-culture progresses in a similar way as under field conditions and that the co-cultures are an adequate biotechnological tool to study this pathology and to easily assess different approaches viewing to understand and combat the CRKN infection mechanism.

\section{Materials and methods}

Establishment of Solanum tuberosum hairy root cultures

Solanum tuberosum cv. Desiree tubers were obtained locally, and prepared for inoculation using the methodology adapted from Kumar and Forrest (1990). The tubers were washed with commercial detergent (10 drops per $100 \mathrm{~mL}$ distilled water) and surface sterilized by immersion in ethanol $96 \%$ (Merck KGaA, Germany) for $10 \mathrm{~min}$. Under asepsis, the potatoes were subsequently rinsed three times in $100 \mathrm{~mL}$ ultrapure sterile water, the peripheral portion removed, approximately $2 \mathrm{~cm}$ inwards from the surface, and the central piece divided into approx. $0.5 \mathrm{~cm}$ thick segments.

HR were induced by inoculation of the aseptic potato segments with $R$. rhizogenes A4 strain [according to new taxononomic revisions (Bull et al. 2010), R. rhizogenes (Riker et al. 1930). Young et al. (2001a) is the most recent synonym of Agrobacterium rhizogenes (Riker et al. 1930) Conn 1942] carrying the gus reporter gene co-integrated in the Ri plasmid and driven by a double $35 \mathrm{~S}$ promoter (A4pRiA4::70GUS), using the methodology described by Santos et al. (1998). The potato segments were wounded with a scalpel previously dipped in an overnight grown $R$. rhizogenes suspension, to an $\mathrm{A}_{600}=0.6$, and diluted 1:10 (v/v) in liquid Schenk and Hildebrandt (SH) medium (Schenk and Hildebrandt 1972) supplemented with $30 \mathrm{~g} \mathrm{~L}^{-1}$ sucrose, $\mathrm{pH}=5.6$. After drying on sterile filter paper, for $1 \mathrm{~min}$, the segments were placed on $\mathrm{SH}$ solid medium ( $8 \mathrm{~g} \mathrm{~L}^{-1}$ agar), and co-cultivated with the bacteria for 3 days after which they were transferred to $\mathrm{SH}$ solid medium supplemented with cefotaxime and carbenicillin $\left(150 \mu \mathrm{g} \mathrm{mL}^{-1}\right.$ each), with both medium and antibiotics renewed weekly, for over 3 months. $S$. tuberosum HR were excised and used for propagation in antibiotic-free SH solid medium. After \pm 3 months free from contaminations, HR pieces were transferred to liquid SH medium without growth regulators and antibiotic and maintained on orbital shakers (80 rpm). Under a regular subculturing routine, a portion of the root clump was aseptically removed and transferred monthly to fresh culture medium. In every step the potato cultures were maintained in darkness at $24 \pm 1{ }^{\circ} \mathrm{C}$. Oneyear-old $S$. tuberosum $\mathrm{HR}$ were characterized by evaluation of their structure, growth and production of volatiles compared with potato HR/CRKN co-cultures.

\section{Establishment of $S$. tuberosum HR/M. chitwoodi co-cultures}

Meloidogyne chitwoodi (CRKN) egg masses were handpicked from infected tomato plants as described by Vieira dos Santos et al. (2013). The eggs were extracted with $0.52 \% \quad(\mathrm{v} / \mathrm{v})$ sodium hypochlorite $(\mathrm{NaOCl})$ solution (Hussey and Barker 1973) and the debris removed with a $47 \%$ (w/v) sucrose solution according to McClure et al. (1973). Afterwards, the eggs were sterilized in a $0.05 \%$ (v/v) mercuric chloride $\left(\mathrm{HgCl}_{2}\right)$ solution for $3 \mathrm{~min}$, centrifuged for $2 \mathrm{~min}$ at $500 \mathrm{~g}$, rinsed in sterile water and centrifuged (four times), as above, to remove $\mathrm{HgCl}_{2}$ (adapted from Kumar and Forrest 1990).

Solanum tuberosum HR/CRKN co-cultures were established by adding 100-150 sterilized CRKN eggs to $S$. $t u$ berosum HR cultures after 14 days of growth on solid SH medium. Co-cultures were maintained in darkness at $24 \pm 1{ }^{\circ} \mathrm{C}$ for over 2 months, to ensure the completion of the nematode life cycle. Life cycle stages were identified using an inverted microscope Diaphot, Nikon, Japan. Subculturing of the co-cultures was performed every 4 weeks, by aseptically transferring a portion of the root clump to fresh solid SH medium, and maintained as described above. After approx. 6 months, root clumps were transferred to liquid SH medium and maintained in darkness at $24 \pm 1{ }^{\circ} \mathrm{C}$, on an orbital shaker ( $80 \mathrm{rpm})$, with monthly subculturing by refreshment of the culture medium over 3 months. Then, a portion of the root clump was removed and transferred to fresh liquid SH culture medium. The length of 30 ramdomly selected HR/CRKN galls, J2, and males as well as the length/width of 30 randomly selected HR/CRKN eggs and adult females were measured using a stage micrometer calibrated eyepiece reticle. Similar to $S$. tuberosum HR, oneyear-old $S$. tuberosum HR/CRKN co-cultures were characterized by evaluation of their structure, growth and production of volatiles. Besides this initial infection stage, no further external nematodes were added to the co-culture, given that it would influence the natural co-culture equilibrium, which intends to mimic natural conditions.

\section{Characterization of $S$. tuberosum HR and S. tuberosum HR/CRKN co-culture structure}

Solanum tuberosum HR and galls, from selected co-cultures with at least one-year of in vitro culture, were collected from solid $\mathrm{SH}$ medium, for scanning electron 
microscopy (SEM), and from liquid SH medium for light microscopy (LM). S. tuberosum HR and galls were prepared for SEM following the methodology described by Figueiredo and Pais (1994). Samples were fixed with $1.5 \%$ (v/v) glutaraldehyde in $0.05 \mathrm{M}$ sodium cacodylate buffer, $\mathrm{pH} 7.0$ for $45 \mathrm{~min}$ at room temperature. After 1-2 min under vacuum $(26 \mathrm{~mm} \mathrm{Hg}, 3.46 \mathrm{kPA})$, the fixative was substituted with $3 \%$ glutaraldehyde in $0.1 \mathrm{M}$ sodium cacodylate buffer, $\mathrm{pH} 7.0$ for $2 \mathrm{~h}$ at room temperature. The material was rinsed thoroughly in the same buffer, postfixed with a $2 \%$ osmium tetroxide aqueous solution $\left(\mathrm{OsO}_{4}\right)$ for $2 \mathrm{~h}$ at room temperature, dehydrated in a graded acetone series and critical point dried in a Polaron E 3500. Dried specimens were mounted on stubs, coated with gold in a Polaron E 5350. Observations were carried out on a JEOL T220 SEM (JEOL Ltd., Tokyo, Japan) at $15 \mathrm{kV}$.

For LM, galls excised from S. tuberosum HR/CRKN cocultures grown in liquid $\mathrm{SH}$ medium were fixed with glutaraldehyde $2.5 \%(\mathrm{v} / \mathrm{v})$ in $0.1 \mathrm{M}$ sodium phosphate buffer, $\mathrm{pH}$ 7.2. Samples were kept in fixative under vacuum at room temperature for $20 \mathrm{~min}$, followed by $24-48 \mathrm{~h}$ at $4{ }^{\circ} \mathrm{C}$. The material was then washed in the fixative buffer, dehydrated in a graded ethanol series, and embedded in Leica historesin ${ }^{\circledR}$ according to Ascensão et al. (2005). Longitudinal and cross sections ( $4 \mu \mathrm{m}$ thick) were sequentially stained with periodic acid-Schiff's (PAS) reagent/Toluidine Blue O (Feder and O'Brien 1968) for polysaccharides and general histology. Observations were made under a Leica DM-2500 microscope (Leica Microsystems CMS GmbH, Wetzlar. Germany). The images were recorded digitally using a Leica DFC-420 camera (Leica Microsystems Ltd., Heerbrugg, Switzerland) and the Leica Application Suite software (version 2.8.1).

Time-course characterization of $S$. tuberosum HR and $S$. tuberosum HR/CRKN co-culture growth, nematode population density and production of volatiles

Erlenmeyer flasks with $100 \mathrm{~mL}$ SH medium were aseptically inoculated with $1 \mathrm{~g}$ fresh weight (FW) of S. tuberosum HR or $S$. tuberosum HR/CRKN kept under routine subculture, and maintained as above. Growth, nematode population density in the culture medium and production of volatiles were evaluated at inoculation time ( $\mathrm{t} 0)$ and weekly for 7 weeks. Two independent experiments were conducted separately, for each in vitro culture, and two replicates of each flask were used in each experiment. The data shown were calculated as mean values of all experiments.

Growth of in vitro cultures

Solanum tuberosum HR and S. tuberosum HR/CRKN co-cultures growth were evaluated by the dissimilation method, a non-invasive method that relates growth with energy-consumption of the cells (Schripsema et al. 1990), and by measuring the FW and DW. FW determination was performed after blotting the total HR cultures or HR/ CRKN co-culture clumps on filter paper to remove excess culture medium. For dry weight (DW) calculation, samples from the clumps were frozen for $24 \mathrm{~h}$ followed by freezedrying for 2 days, in an Alpha I-5 (Martin Christ GmbH, Osterode, Germany) apparatus, at $0.1 \mathrm{mbar}$ and $-42{ }^{\circ} \mathrm{C}$. The total DW was based on the relation between FW and DW determination after freeze-drying. Specific growth rate $(\mu)$ in wt $\mathrm{L}^{-1}$ day $^{-1}$ and doubling time $(\mathrm{dt})$ in days were determined for FW and DW growth curves, between the 4th and 7th day, using the formulae according to Kondo et al. (1989) and Mehrara et al. (2007): $\mu=\left(\log _{\mathrm{e}} \mathrm{X}-\right.$ $\left.\log _{\mathrm{e}} \mathrm{X}_{0}\right) / \mathrm{t} ; \mathrm{dt}=\left(\log _{\mathrm{e}} 2\right) / \mu$, where $\mathrm{X}_{0}$ is the initial weight, $\mathrm{X}$ is the final weight and $\mathrm{t}$ is the time between weighings. The remaining $S$. tuberosum $\mathrm{HR}$ or $S$. tuberosum HR/ CRKN co-cultures were kept at $-20{ }^{\circ} \mathrm{C}$ until volatiles were extracted.

\section{Nematode population density in co-cultures medium}

Population of CRKN ( $\mathrm{J} 2$ and males) in the liquid medium was evaluated by sampling $100 \mu \mathrm{L}$ aliquots of each culture flask at each time-point. Three replicates of each flask were used for counts. Number of dead and live nematodes was recorded using an inverted microscope.

\section{Isolation of volatiles from in vitro cultures}

Constitutive volatiles as well as those induced by the phytoparasite were isolated from the in vitro cultures by distillation-extraction, for $3 \mathrm{~h}$, using a Likens-Nickerson type apparatus (Likens and Nickerson 1964). Distillation was run at $3 \mathrm{~mL} \min ^{-1}$ rate, using in-lab distilled $n$-pentane $(50 \mathrm{~mL})$ (Honeywell Riedel-de Haën, Hanover, Germany) as organic solvent. The volatiles recovered in distilled $n$-pentane were concentrated, at room temperature, under reduced pressure on a rotary evaporator (Yamato, Hitec RE-51). After collection in a vial, the volatiles were concentrated to a minimum volume under nitrogen flux, at room temperature. The volatile oils were stored at $-20{ }^{\circ} \mathrm{C}$ in the dark until analysis.

\section{Analysis of volatiles from in vitro cultures}

Volatiles were analyzed by gas chromatography (GC), for component quantification, and GC coupled to mass spectrometry (GC-MS) for component identification. Gas chromatographic analyses were performed using a Perkin Elmer Autosystem XL gas chromatograph (Perkin Elmer, Shelton, CT, USA) equipped with two flame ionization 
detectors (FIDs), a data handling system, and a vaporizing injector port into which two columns of different polarities were installed: a DB-1 fused-silica column $(30 \mathrm{~m} \times 0.25 \mathrm{~mm}$ i.d., film thickness $0.25 \mu \mathrm{m}$; J \& W Scientific Inc., Rancho Cordova, CA, USA) and a DB$17 \mathrm{HT}$ fused-silica column $(30 \mathrm{~m} \times 0.25 \mathrm{~mm}$ i.d., film thickness $0.15 \mu \mathrm{m}$; J \& W Scientific Inc., Rancho Cordova, CA, USA). Oven temperature was programmed to increase from 45 to $175^{\circ} \mathrm{C}$, at $3{ }^{\circ} \mathrm{C} / \mathrm{min}$ increments, then up to $300{ }^{\circ} \mathrm{C}$ at $15{ }^{\circ} \mathrm{C} / \mathrm{min}$ increments, and finally held isothermal for $10 \mathrm{~min}$. Gas chromatographic settings were as follows: injector and detectors temperatures, 280 and $300{ }^{\circ} \mathrm{C}$, respectively; carrier gas, hydrogen, adjusted to a linear velocity of $30 \mathrm{~cm} / \mathrm{s}$. The samples were injected using a split sampling technique, ratio 1:50. The volume of injection was $0.1 \mu \mathrm{L}$ of a pentane-oil solution (1:1). The percentage composition of the oils was computed by the normalization method from the GC peak areas, calculated as a mean value of two injections from each volatile oil, without response factors.

The GC-MS unit consisted of a Perkin Elmer Autosystem XL gas chromatograph, equipped with DB-1 fusedsilica column $(30 \mathrm{~m} \times 0.25 \mathrm{~mm}$ i.d., film thickness $0.25 \mu \mathrm{m}$; J \& W Scientific, Inc., Rancho Cordova, CA, USA) interfaced with Perkin-Elmer Turbomass mass spectrometer (software version 4.1, Perkin Elmer). GC-MS settings were as follows: injector and oven temperatures were as above; transfer line temperature, $280{ }^{\circ} \mathrm{C}$; ion source temperature, $220{ }^{\circ} \mathrm{C}$; carrier gas, helium, adjusted to a linear velocity of $30 \mathrm{~cm} / \mathrm{s}$; split ratio, 1:40; ionization energy, $70 \mathrm{eV}$; scan range 40-300 $\mu \mathrm{m}$; scan time, $1 \mathrm{~s}$. The identity of the components was assigned by comparison of their retention indices relative to $\mathrm{C}_{8}-\mathrm{C}_{25} n$ alkane indices, and GC-MS spectra from a laboratory made library based upon the analyses of reference oils, laboratory-synthesized components, and commercial available standards.

\section{Results and discussion}

Establishment of Solanum tuberosum hairy root cultures

Solanum tuberosum HR were established on solid $\mathrm{SH}$ medium (Schenk and Hildebrandt 1972), after inoculation of aseptic potato segments with $R$. rhizogenes A4 strain. Approximately 2 weeks after infection, numerous HR were observed at the inoculation sites (Fig. 1a). Following establishment of the HR in liquid medium without antibiotics and growth regulators (Fig. 1b), potato HR were highly branched with the typical "rooty" phenotype (Fig. 1c, d). Structure, growth and volatile analyses were performed after approx. 12 months in culture with routine subculture.

\section{Establishment of $S$. tuberosum HR/CRKN co-cultures}

Solanum tuberosum HR/CRKN co-cultures were obtained by adding surface-sterilized nematode eggs to the HR cultures grown on solid SH medium. The first J2 appeared approximately 2 days after inoculation (DAI) and were seen on the vicinity of the transgenic roots (Fig. 1e). Within approximately 10 days, root tip enlargement was observed and galls and immature females were observed 25 DAI (Fig. 1f, g). Adult females in the galls were detected approximately 45 DAI followed by the production of the gelatinous matrix and eggs (Fig. 1h).

This species is known to cause numerous small, pimplelike, galls that are not always evident, without secondary roots emerging from them. Symptoms caused by $M$. chitwoodi vary according to host, nematode population density and environmental conditions (Perry et al. 2009).

\section{Characterization of $S$. tuberosum $\mathrm{HR}$ and $S$. tuberosum HR/CRKN co-cultures}

\section{Structure of in vitro cultures}

Hairy roots, grown on solid $\mathrm{SH}$ medium, showed the characteristic anatomy of primary root growth structuresa single-layered epidermis with a thin cuticle and numerous lateral hairs, a cortex with few cell layers, an endodermis and a pericycle surrounding the vascular cylinder (Fig. 1c, d). This typical primary root structure has been commonly reported for other HR, such as those of Pimpinella anisum (Santos 1997), Levisticum officinale (Costa 2005) and Anethum graveolens (Geraldes 2010). However, S. tuberosum HR, with an average diameter of $295 \pm 22 \mu \mathrm{m}$, differed from the previous examples by being slightly thinner. According to Verdejo et al. (1988), who studied the reproduction of $M$. javanica in several HR systems, thin roots that grow at moderate rates into the agar and produce many secondary roots could support high nematode reproduction. Thus, $S$. tuberosum HR seemed to have adequate characteristics for $M$. chitwoodi development and reproduction.

In $S$. tuberosum HR/CRKN co-cultures, the HR diameter did not show substantial differences $(304 \pm 38 \mu \mathrm{m})$ compared with $S$. tuberosum HR $(295 \pm 22 \mu \mathrm{m})$, except where root galls were formed $(1375 \pm 260 \mu \mathrm{m})$. The formation of galls was more frequent in roots that grew inside the agar than on the agar surface, suggesting that the location of roots affected nematode penetration and reproduction. A similar observation was made by Verdejo et al. (1988). 

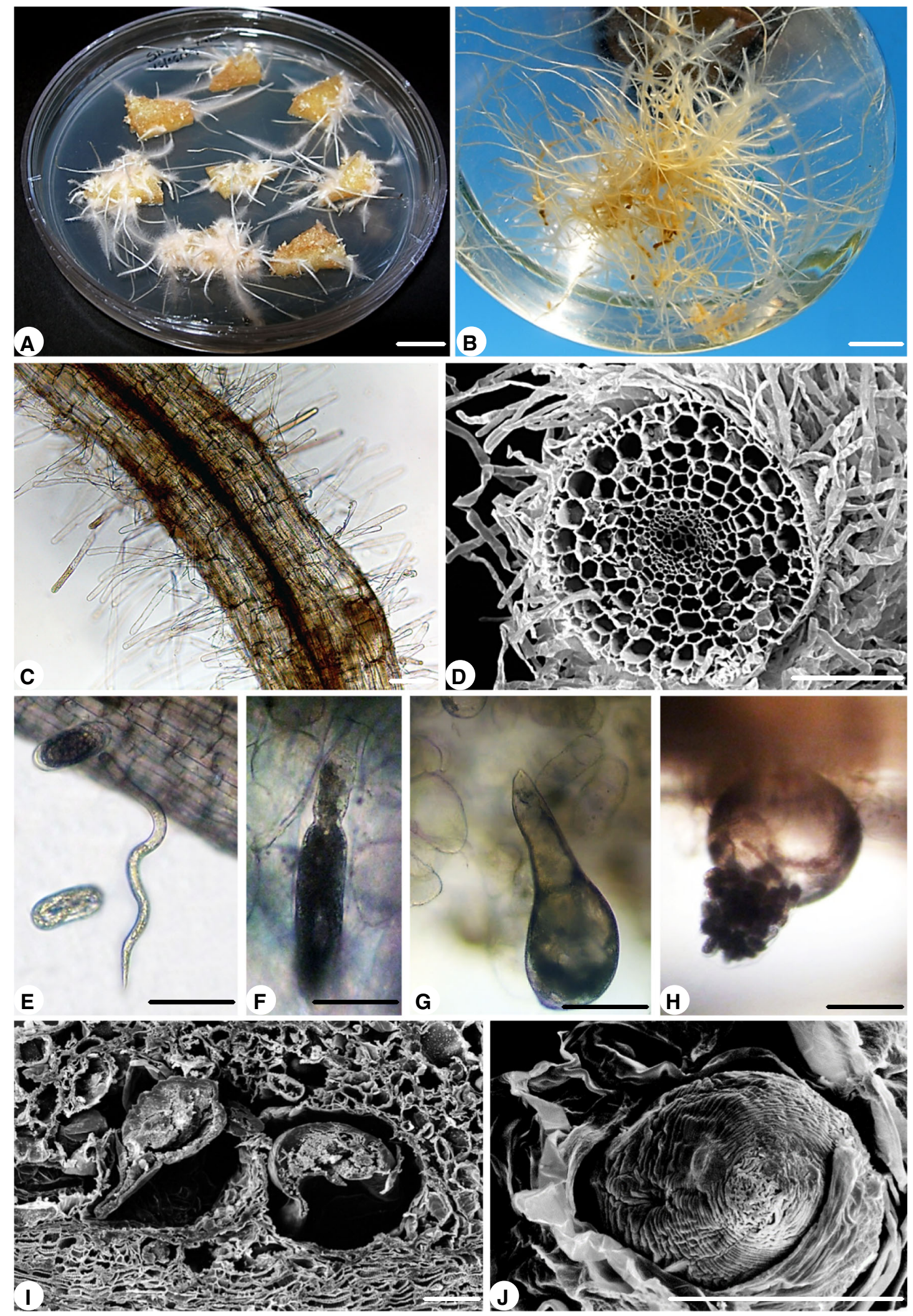
4Fig. 1 Solanum tuberosum hairy (HR) roots and S. tuberosum HR/ Meloidogyne chitwoodi (CRKN) co-cultures. a, b $S$. tuberosum HR cultures grown on solid and liquid Schenk and Hildebrandt (1972) SH medium, respectively. $\mathbf{c}-\mathbf{j}$ Light and scanning electron microscopy micrographs of $S$. tuberosum HR (c, d) and $S$. tuberosum HR/CRKN co-cultures, grown on solid SH medium $(\mathbf{e}-\mathbf{h}, \mathbf{i}, \mathbf{j})$. Note the primary tissues of the root $(\mathbf{c}, \mathbf{d})$, the second-stage juvenile $(\mathbf{e})$, females in different developmental stages (f, $\mathbf{g}$ ) and an adult female with egg mass (h). Pear-shaped females with the head embedded in the periphery of the vascular tissue and the female perineal ridge pattern in $\mathbf{i}$ and $\mathbf{j}$, respectively. Scale bars $1 \mathrm{~cm}(\mathbf{a}, \mathbf{b}), 100 \mu \mathrm{m}(\mathbf{c}-\mathbf{j})$

Scanning electron micrographs (SEM) of co-culture root galls showed CRKN adult pear-shaped females (Fig. 1i, j). Morphometrics of CRKN adult females width/length $(313 \pm 41 / 488 \pm 33 \mu \mathrm{m})$, males length $(950 \pm 91 \mu \mathrm{m})$, $\mathrm{J} 2$ length $(336 \pm 12 \mu \mathrm{m})$ and eggs width/length $(33 \pm 3 /$ $88 \pm 7 \mu \mathrm{m})$ were consistent with the original description of Golden et al. (1980): adult female width/length (344-518/430-740 $\mu \mathrm{m})$; male length $(887-1268 \mu \mathrm{m}) ; \mathrm{J} 2$ length (336-417 $\mu \mathrm{m})$; egg width/length (40-46/ 79-92 $\mu \mathrm{m})$.

In response to signals from the infective stage, root cells from vascular cylinder, adjacent to the head of the nematode, redifferentiated into hypertrophied cells, giant cells (Fig. 2a$\mathrm{d}$, asterisks). These specialized cells were easily distinguished from their neighbouring cells by the increased volume, dense cytoplasm, numerous nuclei and small vacuoles. Another important characteristic feature of these metabolically active cells was the development of cell wall ingrowths (Fig. 2b, c, arrows). Giant cells are typical transfer cells that are involved in rapid solute transport from the contiguous xylem elements to the nematodes, supplying nutrients to nematode development and reproduction (Berg and Taylor 2009). Mature females secrete, at the posterior end, a material that stained dark pink with Periodic acid-Schiff stain (PAS), revealing a polysaccharide nature (Fig. 2e, f). This gelatinous matrix, embedding the eggs layed by females, was usually deposited on the surface of galled roots (Fig. 2g). The root-knot anatomy observed in this study was similar to that induced by RKN described by Berg and Taylor (2009).

Although previous studies addressed the morphology and anatomy of Meloidogyne genus infection and disease progression, the novelty of the present work is that it establishes and characterizes a plant/nematode co-culture as a biotechnological testing system that is reproducible and easy to use, as a tool to evaluate plant/nematode interactions in an in vitro culture system that mimics what occurs in natural conditions.

\section{Growth of in vitro cultures}

A 7 weeks' time-course study was performed to assess potato HR and $S$. tuberosum HR/CRKN co-culture growth, in liquid SH medium, by measuring FW and DW and using the dissimilation method. Compared to $S$. tuberosum HR cultures, $S$. tuberosum HR/CRKN co-cultures showed similar colour, as no browning was induced by the combined growth with M. chitwoodi. Aditionally, S. tuberosum HR growth was not influenced by the presence of the nematode (Fig. 3a, b).

This resemblance in growth profile was supported by the determination of specific growth rate and doubling time based on FW and DW growth curves. Specific growth rates ( $\mu$ ) for $S$. tuberosum HR cultures and $S$. tuberosum HR/ CRKN co-cultures were 0.3 and $0.2 \mathrm{~g} \mathrm{FW} \mathrm{L}^{-1}$ day $^{-1}$, respectively, with doubling times (dt) of 2.6 and 3.0 days on FW basis and 0.3 and $0.2 \mathrm{DW} \mathrm{L}^{-1}$ day $^{-1}$ with dt of 2.5 and 3.5 days, on a DW basis. These values were within the expected for HR cultures. Literature average growth rate for HR ranged from 0.1 to $2.0 \mathrm{~g} \mathrm{~W} \mathrm{~L}^{-1}$ day $^{-1}$ (OksmanCaldentey and Hiltunen 1996) and 1-11 days dt (Maldonado-Mendoza et al. 1993; Dhakulkar et al. 2005), being largely dependent on a high rate of linear extension, lateral branching and secondary increase in root diameter (Figueiredo et al. 2006).

Notwithstanding small variations in growth, probably due to variations in development stages of $S$. tuberosum HR starting inoculum, the outline of the growth curves was identical to those previously observed with Anethum graveolens (Faria et al. 2009) or Levisticum officinale HR (Nunes et al. 2009).

Potato HR growth seemed unaffected by the nematode which may be due to the constant optimal temperature and nutrient availability under in vitro culture conditions, and, as reported by Perry et al. (2009), a plant may be infected and still not show clear changes in growth and productivity. Santo and O'Bannon (Santo and O'Bannon 1981) analysed differences in root weight and RKN reproduction, at different temperatures, in $S$. tuberosum roots inoculated with two starting inocula. At $25^{\circ} \mathrm{C}$, a tenfold increase in the starting inoculum induced an almost threefold decrease in root weight, and lead to the production of more CRKN eggs. In future experiments using this biotechnological model system, it would be advantageous to test different CRKN inoculum levels in order to determine the influence of this parameter on the co-cultures growth.

\section{Nematode population density in co-culture medium}

The number of $M$. chitwoodi motile forms ( $\mathrm{J} 2$ and males) in the medium was quantified at different time-points, every week during 7 weeks, as an indicator of the nematode population density. Twenty one days after inoculation, the number of nematodes increased ( \pm 44 nematodes $\mathrm{mL}^{-1}$ ), concomitant with the progressive growth deceleration of the co-culture, followed by a slight decrease and then another increase $\left( \pm 77\right.$ nematodes $\left.\mathrm{mL}^{-1}\right)$ 


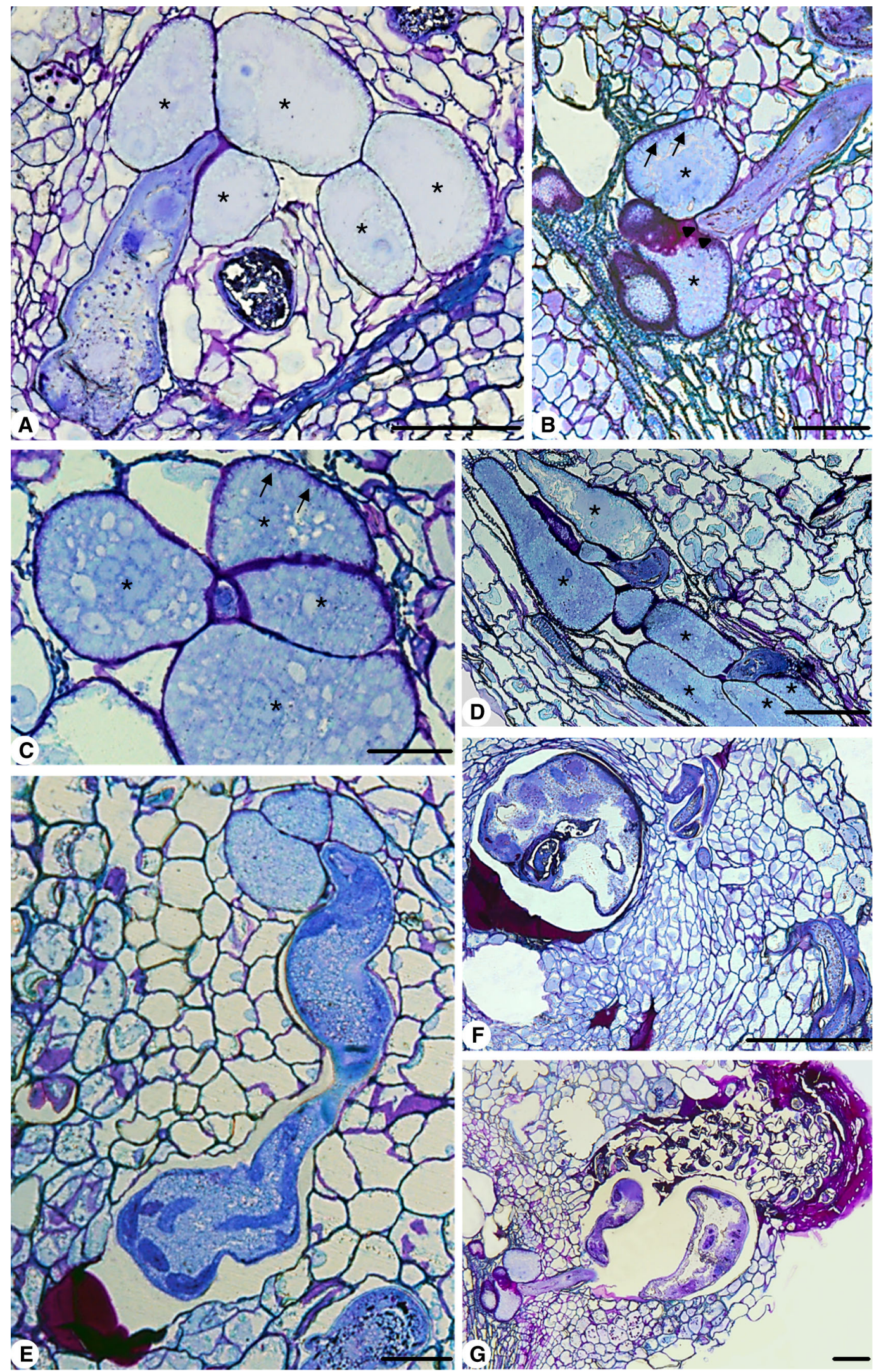


4Fig. 2 Light micrographs of historesin galled root sections from $S$. tuberosum HR/CRKN co-cultures in liquid Schenk and Hildebrandt (1972) SH medium, stained with Periodic Acid-Schiff's (PAS)/ Toluidine Blue O. a, b Females feeding on a group of prominent giant cells (asterisks). Note in b the nematode head embedded in the periphery of the vascular tissue (arrowheads) and the giant cell wall ingrowths (arrows). c Detail of the giant cells with dense cytoplasm, small vacuoles, numerous nuclei and cell wall ingrowths (arrows). d Giant cells and interspersed vascular elements are apparent. ef Longitudinal and cross sections of mature females revealing a pink PAS-positive exudate near their posterior ends. g Eggs embedding in a polysaccharidic matrix are observed on the surface of galled roots. Scale bars $100 \mu \mathrm{m}$

until the end of the sampling time-points, in the co-culture stationary growth phase (Fig. 3a). The first peak may be due to $\mathrm{J} 2$ hatching from eggs produced by females present in the inoculated co-culture root clump (1st generation), and the second peak to the second generation of nematodes (Fig. 3a). Second generation of nematodes was originated from co-culture new infections and the production of new mature females, whose hatched juveniles contributed to the second peak in the nematode number.
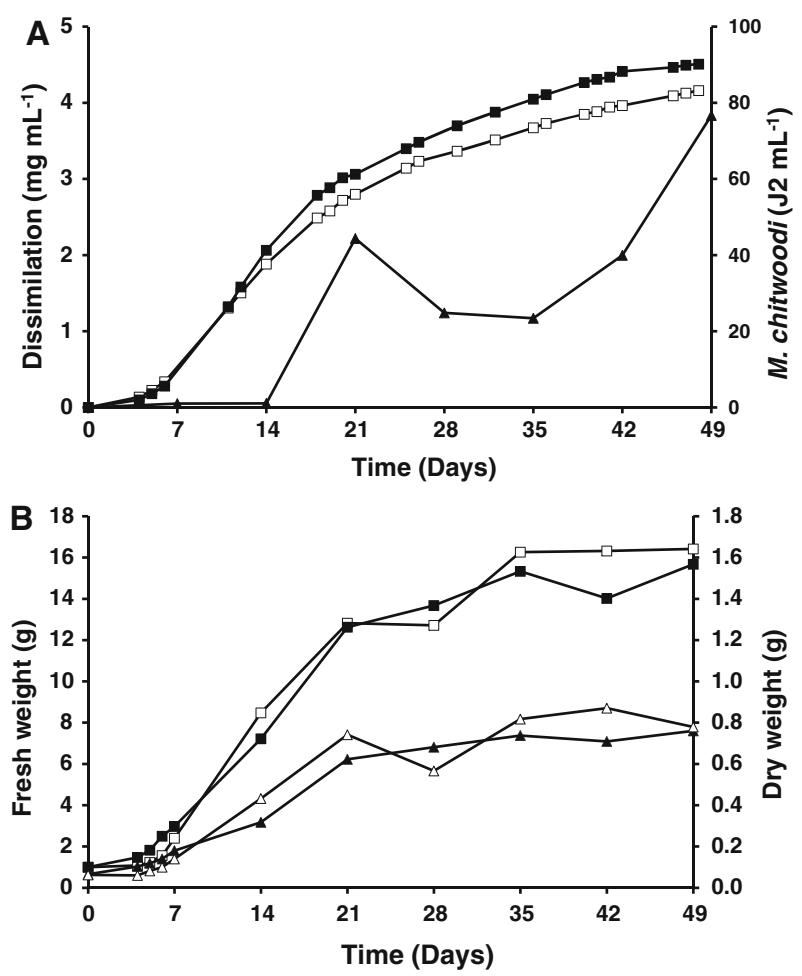

Fig. 3 a Dissimilation growth curves of Solanum tuberosum hairy roots (HR) (open square) and $S$. tuberosum HR/Meloidogyne chitwoodi (CRKN) co-cultures (filled square), and number of nematodes in the culture medium (filled triangle). b Fresh (square symbols) and dry weight (triangle symbols) growth curves of $S$. tuberosum HR and $S$. tuberosum HR/CRKN co-cultures. Fresh weight growth curves: $S$. tuberosum HR (open square) and co-cultures (filled square). Dry weight growth curves: $S$. tuberosum HR (open triangle) and co-cultures (filled triangle)
Continuous subculturing of these co-cultures in $\mathrm{SH}$ medium reproduced the same growth pattern leading to an equilibrium between nematode infection and development and HR growth, making possible the maintenance of this co-culture. Pak et al. (2009) analysed M. incognita infection and development in Cucumis melo HR grown in three culture media and found that the greatest number of egg masses were obtained on HR cultured in SH medium. Culture medium appears to contribute to the success in the establishment and maintenance of $\mathrm{HR} /$ nematode cocultures.

Production of volatiles during in vitro culture

Thirty one compounds were identified in the constitutive volatiles isolated from $S$. tuberosum HR. The same compounds were identified in the volatiles isolated from $S$. tuberosum HR/CRKN co-cultures, maintained under the same growth conditions. The detailed relative amounts of the components identified in the volatiles, isolated from potato in vitro cultures, are listed according to their elution from a DB-1 column (Table 1). A limited number of components, with relative amounts of $0.5-3 \%$ each, could not yet be identified. Together they justify the lower identification attained at time 0 for HR/CRKN co-cultures volatiles. Nevertheless, their relative importance decreases during the time-course study of volatiles in these cultures.

Fatty acids (33-63\%) and the fraction designated by others (23-39\%), since components were neither terpenes nor C13 compounds, and which was mainly composed of non-aromatic alcohols, saturated and unsaturated non-aromatic aldehydes and hydrocarbons, dominated both the constitutive volatiles of the HR and those of the HR/CRKN co-cultures (Table 1). Palmitic acid (37-52\% in the HR and $24-44 \%$ in the co-cultures), $n$-pentadecanal (6-16\% in the HR and 8-22\% in the co-cultures) and linoleic acid (2-16\% in the HR and 4-18\% in the co-cultures) dominated $S$. tuberosum HR and $S$. tuberosum HR/CRKN volatiles. Fatty acid abundance, mostly resulting from cell membrane degradation, is common in constitutive volatile profiles of HR (Faria et al. 2009; Nunes et al. 2009), as well as in plants producing small amount of volatiles.

Although Komaraiah et al. (2003) reported enhanced production of the stress related antimicrobial sesquiterpene phytoalexins rishitin, lubimin, phytuberin and phytuberol, extracted with organic solvents, from elicitor-treated HR cultures of $S$. tuberosum, these compounds were not detected in the present study. These results can be explained by the different responses to elicitors or to CRKN, or that, if produced, the compounds can undergo glycosylation, as observed in L. officinale HR (Nunes et al. 2009), rendering them non-volatile and thus not directly extractable by hydrodistillation. 
Table 1 Percentage composition of the volatiles isolated from Solanum tuberosum HR and S. tuberosum HR/CRKN co-cultures, at the different time-points (t0, inoculation time)

\begin{tabular}{|c|c|c|c|c|c|c|c|c|c|c|c|c|c|c|c|c|c|}
\hline \multirow[t]{3}{*}{ Component } & \multirow[t]{3}{*}{ RI } & \multicolumn{8}{|c|}{ S. tuberosum HR } & \multicolumn{8}{|c|}{ S. tuberosum HR/CRKN } \\
\hline & & \multicolumn{8}{|c|}{ Time (days) } & \multicolumn{8}{|c|}{ Time (days) } \\
\hline & & 0 & 7 & 14 & 21 & 28 & 35 & 42 & 49 & 0 & 7 & 14 & 21 & 28 & 35 & 42 & 49 \\
\hline$n$-Hexanol & 882 & $\mathrm{t}$ & 0.7 & 0.4 & 0.1 & 0.1 & 0.2 & 0.2 & 0.2 & 0.5 & 0.3 & 0.2 & 0.1 & 0.2 & 0.2 & 0.4 & 0.6 \\
\hline 2-Pentyl furan & 973 & 0.2 & 1.4 & 1.4 & 0.5 & 8.1 & 1.1 & 0.5 & 0.3 & 0.8 & 1.7 & 1.9 & 1.1 & 0.9 & 0.9 & 0.7 & 1.0 \\
\hline$\beta$-Myrcene & 975 & 1.1 & 0.6 & 0.9 & 0.4 & 3.1 & 2.6 & 0.3 & 0.6 & 0.4 & 0.5 & 0.9 & 1.0 & 2.8 & 0.6 & 0.5 & 0. \\
\hline Benzyl alcohol & 1000 & 4.2 & 4.4 & 4.9 & 2.8 & 6.9 & 4.3 & 5.4 & 5.4 & 3.3 & 6.0 & 4.7 & 5.7 & 4.6 & 5.6 & 7.4 & 3.0 \\
\hline Benzene acetaldehyde & 1002 & $\mathrm{t}$ & $\mathrm{t}$ & $\mathrm{t}$ & $\mathrm{t}$ & $\mathrm{t}$ & $\mathrm{t}$ & $\mathrm{t}$ & $\mathrm{t}$ & $\mathrm{t}$ & $\mathrm{t}$ & $\mathrm{t}$ & $\mathrm{t}$ & $\mathrm{t}$ & $\mathrm{t}$ & $\mathrm{t}$ & $\mathrm{t}$ \\
\hline$n$-Octanol & 1045 & 0.3 & 0.1 & 0.4 & 0.1 & 0.1 & 0.2 & 0.2 & 0.4 & 0.3 & 0.1 & 0.1 & 0.2 & 0.1 & 0.6 & 0.3 & 0.2 \\
\hline$o$-Guaiacol ${ }^{\mathrm{g}}$ & 1058 & 2.6 & 3.8 & 2.9 & 1.9 & 2.5 & 4.4 & 3.2 & 2.8 & 5.8 & 2.2 & 2.9 & 3.3 & 3.1 & 2.8 & 4.6 & 4.1 \\
\hline 2-Methyl decane & 1058 & 0.2 & $\mathrm{t}$ & 0.1 & 0.1 & $\mathrm{t}$ & $\mathrm{t}$ & 0.1 & 0.1 & $\mathrm{t}$ & 0.3 & $\mathrm{t}$ & $\mathrm{t}$ & $\mathrm{t}$ & 0.1 & $\mathrm{t}$ & 0.5 \\
\hline Phenyl ethyl alcohol & 1064 & 0.5 & 1.1 & 0.4 & 0.3 & 0.5 & 0.5 & 1.7 & 1.6 & 0.3 & 0.3 & 0.4 & 0.5 & 0.4 & 0.3 & 0.6 & 0.2 \\
\hline trans-Pinocarveol & 1106 & $\mathrm{t}$ & 0.3 & 0.1 & 0.1 & 0.1 & 0.1 & 0.1 & 0.1 & 0.1 & 0.1 & 0.3 & 0.2 & 0.1 & 0.3 & 0.1 & 0.3 \\
\hline 3-cis-Nonen-1-ol & 1124 & $\mathrm{t}$ & 0.7 & 0.7 & 1.0 & 0.6 & 1.0 & 0.8 & 0.8 & 2.2 & 0.7 & 1.5 & 0.8 & 1.1 & 1.4 & 1.4 & 2.1 \\
\hline $\begin{array}{l}\text { trans-Mentha-1(7),8-dien- } \\
\text { 2-ol }\end{array}$ & 1159 & 0.2 & 0.4 & 0.1 & 0.1 & 0.1 & 0.1 & $\mathrm{t}$ & $\mathrm{t}$ & 0.2 & 0.1 & 0.3 & 0.1 & 0.1 & 0.1 & 0.1 & 0.3 \\
\hline $\begin{array}{l}\text { 2-trans, 4-trans- } \\
\text { Nonadienal }\end{array}$ & 1184 & 0.4 & 0.7 & 0.2 & 0.3 & 0.1 & 0.2 & 0.1 & 0.2 & 0.4 & 0.2 & 0.3 & 0.3 & 0.2 & 0.1 & 0.1 & 0.3 \\
\hline Carvacrol & 1286 & 1.7 & 1.3 & 1.1 & 0.9 & 0.6 & 1.6 & 1.6 & 1.6 & 2.2 & 2.2 & 0.9 & 1.6 & 1.7 & 1.8 & 2.2 & 2.7 \\
\hline$n$-Tetradecanal & 1596 & 2.0 & 0.8 & 1.2 & 1.4 & 1.3 & 1.8 & 2.1 & 1.6 & 2.2 & 2.7 & 0.8 & 1.0 & 1.2 & 2.4 & 2.7 & 3.6 \\
\hline $\begin{array}{l}\text { cis-Methyl } \\
\text { dehydrojasmonate }\end{array}$ & 1640 & 0.1 & 0.4 & 0.2 & 0.1 & $\mathrm{t}$ & 0.1 & 0.1 & $\mathrm{t}$ & 0.1 & $\mathrm{t}$ & 0.2 & 1.1 & $\mathrm{t}$ & $\mathrm{t}$ & 0.4 & 0.5 \\
\hline$n$-Pentadecanal & 1688 & 11.0 & 5.6 & 9.7 & 12.0 & 9.8 & 15.2 & 16.2 & 13.7 & 11.4 & 8.5 & 7.6 & 8.2 & 9.6 & 17.0 & 15.6 & 21.7 \\
\hline Myristic acid & 1723 & 0.1 & 2.6 & 0.6 & 0.6 & 0.6 & 0.9 & 1.0 & 1.0 & 0.6 & 1.9 & 0.5 & 1.0 & 0.8 & 0.7 & 0.6 & 1.1 \\
\hline Isopropyl decanoate ${ }^{\mathrm{g}}$ & 1753 & 0.8 & 1.5 & 1.0 & 0.8 & 0.5 & 0.2 & 0.4 & 0.4 & 1.4 & 2.0 & 0.9 & 0.6 & 0.5 & 0.4 & 0.3 & 0.1 \\
\hline Pentadecanoic acid ${ }^{\text {bg }}$ & 1776 & 2.2 & 2.3 & 2.5 & 3.1 & 3.0 & 4.2 & 4.1 & 4.2 & 1.5 & 3.4 & 2.2 & 3.9 & 4.4 & 3.5 & 3.7 & 1.6 \\
\hline$n$-Hexadecanol & 1821 & 0.3 & 5.8 & 0.3 & 0.2 & 0.2 & 0.1 & 0.2 & 0.2 & 0.2 & 2.4 & 0.3 & 0.1 & 0.2 & 0.2 & 0.3 & 0.2 \\
\hline$n$-Heptadecanal & 1894 & 0.5 & 1.1 & 0.8 & 0.8 & 0.6 & 0.5 & 0.6 & 0.4 & 0.9 & 2.5 & 0.8 & 0.4 & 0.4 & 0.4 & 0.4 & 0.4 \\
\hline Palmitic acid & 1908 & 51.6 & 35.5 & 36.6 & 44.2 & 37.6 & 36.7 & 37.1 & 39.5 & 23.8 & 39.5 & 37.8 & 43.7 & 42.7 & 38.9 & 38.6 & 32.4 \\
\hline Heptadecanol ally & 1987 & 0.4 & 0.9 & 0.6 & 1.0 & 1.1 & 1.2 & 0.2 & 0.2 & 0.5 & 0.7 & 1.0 & 1.1 & 0.6 & 0.9 & 1.0 & 0.9 \\
\hline Margaric acid ${ }^{\mathrm{dg}}$ & 2032 & 0.5 & 0.5 & 0.2 & 0.2 & 0.2 & 0.3 & 1.8 & 1.9 & 0.1 & 0.4 & 0.1 & 0.2 & 0.3 & 0.2 & 0.2 & $\mathrm{t}$ \\
\hline$n$-Octadecanol & 2071 & 0.1 & 0.2 & 0.1 & 0.1 & $\mathrm{t}$ & $\mathrm{t}$ & 0.1 & 0.1 & $\mathrm{t}$ & 0.3 & 0.1 & $\mathrm{t}$ & 0.1 & 0.7 & $\mathrm{t}$ & $\mathrm{t}$ \\
\hline Phytol acetate & 2047 & $\mathrm{t}$ & $\mathrm{t}$ & $\mathrm{t}$ & $\mathrm{t}$ & $\mathrm{t}$ & $\mathrm{t}$ & $\mathrm{t}$ & $\mathrm{t}$ & $\mathrm{t}$ & $\mathrm{t}$ & $\mathrm{t}$ & $\mathrm{t}$ & $\mathrm{t}$ & $\mathrm{t}$ & $\mathrm{t}$ & $\mathrm{t}$ \\
\hline Linoleic acid ${ }^{\mathrm{e}}$ & 2125 & 1.7 & 9.9 & 16.1 & 12.4 & 6.9 & 6.5 & 7.4 & 8.4 & 6.3 & 11.5 & 18.1 & 10.1 & 8.0 & 6.5 & 3.7 & 5.7 \\
\hline Stearic acid ${ }^{\mathrm{f}}$ & 2149 & 0.6 & 2.6 & 0.5 & 2.4 & 0.8 & 1.8 & 1.0 & 0.9 & 0.8 & 2.8 & 1.5 & 1.4 & 1.7 & 1.0 & 0.5 & 0.6 \\
\hline$n$-Eicosanal & 2200 & $\mathrm{t}$ & 0.5 & $\mathrm{t}$ & 0.1 & 0.1 & 0.2 & 0.2 & 0.2 & 0.1 & 0.2 & 0.1 & 0.1 & 0.1 & 0.2 & 0.1 & 0.1 \\
\hline$n$-Docosanal & 2426 & $\mathrm{t}$ & 0.6 & $\mathrm{t}$ & 0.1 & 0.2 & $\mathrm{t}$ & $\mathrm{t}$ & $\mathrm{t}$ & $\mathrm{t}$ & 0.3 & $\mathrm{t}$ & $\mathrm{t}$ & $\mathrm{t}$ & 0.3 & $\mathrm{t}$ & 0.1 \\
\hline$\%$ Identification & & 83.3 & 85.5 & 83.8 & 88.0 & 85.1 & 85.7 & 86.1 & 86.2 & 66.4 & 93.3 & 86.1 & 87.7 & 85.8 & 87.6 & 86.1 & 85.2 \\
\hline \multicolumn{18}{|l|}{ Grouped components } \\
\hline Monoterpene 1 & & 1. & 0.6 & 0.9 & 0.4 & 3. & 2.6 & 0.3 & 0.6 & 0.4 & 0.5 & 0.9 & 1.0 & 2.8 & 0.6 & 0.5 & 0.9 \\
\hline $\begin{array}{l}\text { Oxygen-containing } \\
\text { monoterpenes }\end{array}$ & & 1.9 & 1.9 & 1.3 & 1.1 & 0.7 & 1.8 & 1.7 & 1.6 & 2.5 & 2.4 & 1.6 & 1.9 & 1.8 & 2.1 & 2.4 & 3.3 \\
\hline C13 compounds & & 0.1 & 0.1 & 0.2 & 0.1 & $\mathrm{t}$ & 0.1 & 0.1 & $\mathrm{t}$ & 0.1 & $\mathrm{l}$ & 0.2 & 1.1 & $\mathrm{t}$ & $\mathrm{t}$ & 0.4 & 0.5 \\
\hline Fatty acids & & 56.7 & 53.3 & 56.5 & 62.8 & 49.0 & 50.2 & 52.3 & 55.8 & 33.1 & 59.5 & 60.1 & 60.3 & 57.9 & 50.8 & 47.2 & 41.4 \\
\hline Others & & 23.5 & 29.4 & 24.9 & 23.6 & 32.3 & 31.0 & 31.8 & 28.2 & 30.3 & 31.0 & 23.3 & 23.4 & 23.2 & 34.1 & 35.7 & 39.1 \\
\hline
\end{tabular}

RI, Lab calculated retention index relative to $\mathrm{C}_{8}-\mathrm{C}_{25} n$-alkanes on the DB-1 column; t, Trace $(<0.05 \%)$

${ }^{\mathrm{a}}$ Tetradecanoic acid, ${ }^{\mathrm{b}}$ Pentadecylic acid, ${ }^{\mathrm{c}}$ Hexadecanoic acid, ${ }^{\mathrm{d}}$ Heptadecanoic acid, ${ }^{\mathrm{e}}$ cis,cis,-9,12-Octadecadienoic acid, ${ }^{\mathrm{f}}$ Octadecanoic acid

g Identification based on mass spectra only 
Other factors, such as increase in nematode load due to prolonged period of culture might result in an altered stress-induced volatile response. Nevertheless, it was not the goal of this study to stress the HR/CRKN co-cultures but, instead, to obtain an equilibrated in vitro co-culture system where variations in volatiles can be easily detected, e.g., after the external application of nematicidals.

To our knowledge, this is the first report on the effect of M. chitwoodi infection on $S$. tuberosum HR production of volatiles. In other studies, Desjardins et al. (1997) identified altered ratios of solavetivone versus total sesquiterpenes in Globodera rostochiensis potato resistant genotypes. Veech (1978) and Khoshkhoo et al. (1994) related the resistance of cotton to $M$. incognita with increased production of terpene aldehydes and according to Edens et al. (1995), the resistance may be linked to the host's ability to perceive nematode infection, as resistant Glycine max plants showed greater expression of genes encoding enzymes from the phenylpropanoid pathway (defense response enzymes) in response to RKN infection while susceptible plants did not.

In conclusion, $S$. tuberosum HR cultures and $S$. tuberosum HR/CRKN co-cultures were successfully established and their structure, growth and volatiles evaluated. The presence of the phytoparasite was not detrimental as both HR cultures and co-cultures showed similar growth and volatile profiles. These results suggest that the established in vitro co-cultures may be used to simulate the CRKN in vivo infection mechanism, making them a suitable biotechnological tool to research the effect of RKN nematotoxic compounds, while determining their effect on, or the biotransformation capacity of the host plant.

Acknowledgments The authors would like to thank Dr Aiden C. Parte (LPSN-bacterio.net), Prof. Dr Leonor Faleiro (Universidade do Algarve, Faculdade de Ciências e Tecnologia, CBV, IBB) and Dr Daniela Pinto (Universidade de Lisboa, Faculdade de Ciências, Laboratório de Biotecnologia e Microbiologia, BioFIG) for providing information on $R$. rhizogenes recent taxonomy; and Dr Rian Stekelenburg from HZPC Holland B.V. for providing potato cultivar nomenclature. Jorge Faria is grateful to the Fundação para a Ciência e a Tecnologia (FCT) for the PhD grant SFRH/BD/43738/2008. This study was partially funded by FCT, under Pest-OE/EQB/LA0023/ 2011 and research contract PTDC/AGR-CFL/117026/2010.

\section{References}

Andrés MF, Gonzáles-Coloma A, Sanz J, Burillo J, Sainz P (2012) Nematicidal activity of essential oils: a review. Phytochem Rev 11:371-390. doi:10.1007/s11101-012-9263-3

Ascensão L, Francisco A, Cotrim H, Pais MS (2005) Comparative structure of the labellum in Ophrys fusca and O. lutea (Orchidaceae). Am J Bot 92:1059-1067. doi:10.3732/ajb.92.7. 1059

Berg RH, Taylor CG (2009) Cell biology of plant nematode parasitism. Plant cell monographs, vol 15. Springer, Berlin
Bonga JM, Durzan DJ (1982) Tissue culture in forestry. Forestry sciences, vol 5. Martinus Nijhoff/Dr W. Junk Publishers, The Hague

Bull CT, De Boer SH, Denny TP, Firrao G, Fischer-Le Saux M, Saddler GS, Scortichini M, Stead DE, Takikawa Y (2010) Comprehensive list of names of plant pathogenic bacteria, 1980-2007. J Plant Pathol 92:551-592

Chitwood DJ (2002) Phytochemical based strategies for nematode control. Annu Rev Phytopathol 40:221-249. doi:10.1146/ annurev.phyto.40.032602.130045

Chitwood DJ (2003) Nematicides. In: Plimmer JR (ed) Encyclopedia of agrochemicals, vol 3. Wiley, New York, pp 1104-1115

Conceição IL, Cunha MJM, Feio G, Correia M, Santos MCV, Abrantes IMO, Santos MSNA (2009) Root-knot nematodes, Meloidogyne spp., on potato in Portugal. Nematology 11:311-313

Costa MM (2005) Raízes transgénicas de Levisticum officinale como sistema modelo para o estudo da produção de voláteis. M.Sc thesis. Faculdade de Ciências da Universidade de Lisboa

Desjardins AE, McCormick SP, Plaisted RL, Brodie BB (1997) Association between solavetivone production and resistance to Globodera rostochiensis in potato. J Agric Food Chem 45:2322-2326. doi:10.1021/jf960830p

Dhakulkar A, Ganapathi TR, Bhargava S, Bapat VA (2005) Induction of hairy roots in Gmelina arborea Roxb. and production of verbascoside in hairy roots. Plant Sci 169:812-818. doi:10.1016/ j.plantsci.2005.05.014

Edens RM, Anand SC, Bolla RI (1995) Enzymes of the phenylpropanoid pathway in soybean infected with Meloidogyne incognita or Heterodera glycines. J Nematol 27:292-303

EPPO (2012) Data sheets on quarantine pests: Meloidogyne chitwoodi. http://www.eppo.int/QUARANTINE/nematodes/Meloido gyne_chitwoodi/MELGCH_ds.pdf. Accessed 15 March 2013

Faria JMS, Nunes IS, Figueiredo AC, Pedro LG, Trindade H, Barroso JG (2009) Biotransformation of menthol and geraniol by hairy root cultures of Anethum graveolens: effect on growth and volatile components. Biotechnol Lett 31:897-903. doi:10.1007/ s10529-009-9934-3

Feder N, O'Brien TP (1968) Plant microtechnique: some principles and new methods. Am J Bot 55:123-142

Ferris H, Carlson HL, Viglierchio DR, Westerdahl BB, Wu FW, Anderson CE, Juurma A, Kirby DW (1993) Host status of selected crops to Meloidogyne chitwoodi. J Nematol 25:849-857

Figueiredo AC, Pais MS (1994) Ultrastructural aspects of the glandular cells from the secretor trichomes and from cell suspension cultures of Achillea millefolium L. ssp. millefolium. Ann Bot-London 74:179-190

Figueiredo AC, Barroso JG, Pedro LG, Scheffer JJC (2006) Potentialities of hairy root cultures for in vitro essential oil production. In: Teixeira da Silva JA (ed) Floriculture, ornamental and plant biotechnology. Global Science Books Ltd., Middlesex. pp 478-486

Geraldes DMA (2010) Biotransformação de monoterpenos por raízes transgénicas de Anethum graveolens. M.Sc. Thesis. Faculdade de Ciências da Universidade de Lisboa

Giri A, Narasu ML (2000) Transgenic hairy roots: recent trends and applications. Biotechnol Adv 18:1-22. doi:10.1016/S07349750(99)00016-6

Golden AM, O'Bannon JH, Santo GS, Finley AM (1980) Description and SEM observations of Meloidogyne chitwoodi n. sp. (Meloidogynidae), a root-knot nematode on potato in the Pacific Northwest. J Nematol 12:319-327

Griffin GD (1985) Host-Parasite relationship of Meloidogyne chitwoodi on potato. J Nematol 17:395-399

Hansen E, Harper G, McPherson MJ (1996) Differential expression patterns of the wound-inducible transgene wun1-uidA in potato roots following infection with either cyst or root knot nematodes. Physiol Mol Plant P 48:161-170. 10.1006/pmpp.1996.0014 
Hussey RS, Barker KR (1973) A comparison of methods of collecting inocula of Meloidogyne spp., including a new technique. Plant Dis Rep 57:1025-1028

Khoshkhoo N, Hedin PA, McCarty JC (1994) Terpenoid aldehydes in root-knot nematode susceptible and resistant cotton plants. J Agric Food Chem 42:204-208. doi:10.1021/jf00037a037

Komaraiah P, Reddy GV, Reddy PS, Raghavendra AS, Ramakrishna SV, Reddanna P (2003) Enhanced production of antimicrobial sesquiterpenes and lipoxygenase metabolites in elicitor-treated hairy root cultures of Solanum tuberosum. Biotechnol Lett 25:593-597. doi:10.1023/A:1023038804556

Kondo O, Honda H, Taya M, Kobayashi T (1989) Comparison of growth properties of carrot hairy root in various bioreactors. Appl Microbiol Biotechnol 32:291-294. doi:10.1007/BF00184976

Kumar A, Forrest JMS (1990) Reproduction of Globodera rostochiensis on transformed roots of Solanum tuberosum cv. Desiree. J Nematol 22:395-398

Li J, Todd TC, Lee J, Trick HN (2011) Biotechnological application of functional genomics towards plant-parasitic nematode control. Plant Biotechnol J 9:936-944. doi:10.1111/j.1467-7652.2011. 00601.x

Likens ST, Nickerson GB (1964) Detection of certain Hop oil constituents in brewing products. Am Soc Brew Chem Proc 5:13-19

Maheshwari R (1991) Applications of plant tissue and cell culture in the study of physiology of parasitism. Proc Indian Acad Plant Sci 69:152-172

Maldonado-Mendoza IE, Ayora-Talavera T, Loyola-Vargas VM (1993) Establishment of hairy root cultures of Datura stramonium. Plant Cell Tiss Org 33:321-329. doi:10.1007/BF02319018

McClure MA, Kruk TH, Misaghi I (1973) A method for obtaining quantities of clean Meloidogyne eggs. J Nematol 5:230

Mehrara E, Forssell-Aronsson E, Ahlman H, Bernhardt P (2007) Specific growth rate versus doubling time for quantitative characterization of tumor growth rate. Cancer Res 67:3970-3975. doi:10.1158/ 0008-5472.CAN-06-3822

Mitkowski NA, Abawi GS (2002) Monoxenic maintenance and reproduction of root-knot nematode (Meloidogyne hapla) on multiple-species in vitro root culture systems. Plant Cell Rep 21:14-23. doi:10.1007/s00299-002-0468-6

Mojtahedi H, Santo GS, Wilson JH (1988) Host tests to differentiate Meloidogyne chitwoodi races 1 and 2 and M. hapla. J Nematol 20:468-473

Nagy R, Karandashov V, Chague V, Kalinkevich K, Tamasloukht M, Xu G, Jakobsen I, Levy A, Amrhein N, Bucher M (2005) The characterization of novel mycorrhiza-specific phosphate transporters from Lycopersicon esculentum and Solanum tuberosum uncovers functional redundancy in symbiotic phosphate transport in solanaceous species. Plant J 42:236-250. doi:10.1111/j. 1365-313X.2005.02364.X

Nunes IS, Faria JMS, Figueiredo AC, Pedro LG, Trindade H, Barroso JG (2009) Menthol and geraniol biotransformation and glycosylation capacity of Levisticum officinale hairy roots. Planta Med 75:387-391. doi:10.1055/s-0028-1112217

O'Bannon JH, Santo GS, Nyczepir AP (1982) Host range of the Columbian root-knot nematode. Plant Dis 66:1045-1048
OEPP/EPPO (2009) Meloidogyne chitwoodi and Meloidogyne fallax. EPPO Bull 39:5-17

Oksman-Caldentey KM, Hiltunen R (1996) Transgenic crops for improved pharmaceutical products. Field Crop Res 45:57-69. doi:10.1016/0378-4290(95)00059-3

Pak HK, Sim JS, Rhee Y, Ko HR, Ha SH, Yoon MS, Kang CH, Lee S, Kim YH, Hahn BS (2009) Hairy root induction in Oriental melon (Cucumis melo) by Agrobacterium rhizogenes and reproduction of the root-knot nematode (Meloidogyne incognita). Plant Cell Tiss Organ Cult 98:219-228. doi:10.1007/ s11240-009-9556-4

Perry RN, Moens M, Starr JL (2009) Root-knot nematodes. CAB International, UK

Santo GS, O'Bannon JH (1981) Effect of soil temperature on the pathogenicity and reproduction of Meloidogyne chitwoodi and M. hapla on Russet Burbank potato. J Nematol 13:483-486

Santo GS, Finley AM, Golden AM (1980) Occurrence and host range of a new root-knot nematode (Meloidogyne chitwoodi) in the Pacific Northwest. Plant Dis 64:951-952. doi:10.1094/PD-64-951

Santos PM (1997) Raízes transformadas de Pimpinella anisum: morfologia, crescimento e caracterização da produção de voláteis. M.Sc. Thesis. Faculdade de Ciências da Universidade de Lisboa

Santos PM, Figueiredo AC, Oliveira MM, Barroso JG, Pedro LG, Deans SG, Younus AKM, Scheffer JC (1998) Essential oils from hairy root cultures and from fruits and roots of Pimpinela anisum. Phytochemistry 48:455-460. doi:10.1016/S0031-9422(98)00022-3

Schenk UR, Hildebrandt AC (1972) Medium and techniques for induction and growth of monocotyledonous and dicotyledonous plant cell cultures. Can J Bot 50:199-204. doi:10.1139/b72-026

Schripsema J, Meijer AH, Iren F, ten Hoopen HJG, Verpoorte R (1990) Dissimilation curves as a simple method for the characterization of growth of plant cell suspension cultures. Plant Cell Tiss Org 22:55-64. doi:10.1007/BF00043699

Valancin A, Srinivasan B, Rivoal J, Jolicoeur M (2013) Analyzing the effect of decreasing cytosolic triosephosphate isomerase on Solanum tuberosum hairy root cells using a kinetic-metabolic model. Biotechnol Bioeng 110:924-935. doi:10.1002/bit.24747

Veech JA (1978) An apparent relationship between methoxy-substituted terpenoid aldehydes and the resistance of cotton to Meloidogyne incognita. Nematologica 24:81-87. doi:10.1163/ $187529278 X 00092$

Verdejo S, Jafee BA, Mankau R (1988) Reproduction of Meloidogyne javanica on plant roots genetically transformed by Agrobacterium rhizogenes. J Nematol 20:599-604

Vieira dos Santos MC, Curtis RHC, Abrantes IMO (2013) Effect of plant elicitors on the reproduction of the root-knot nematode Meloidogyne chitwoodi on susceptible hosts. Eur J Plant Pathol 136:193-202. doi:10.1007/s10658-012-0155-6

Wiśniewska A, Dabrowska-Bronk J, Szafranski K, Fudali S, Swiecicka M, Czarny M, Wilkowska A, Morgiewicz K, Matusiak J, Sobczak M, Filipecki M (2013) Analysis of tomato gene promoters activated in syncytia induced in tomato and potato hairy roots by Globodera rostochiensis. Transgenic Res 22:557-569. doi:10.1007/s11248-012-9665-4 